\title{
Report
}

\section{Incidental Findings of the Lumbar Spine at MRI in Patients Diagnosed with Discopathy}

\author{
Mohammad Sobhan ${ }^{1}$, Majid Samiee ${ }^{1}$, Yadollah Asgari ${ }^{2}$, Mansour Ahmadi ${ }^{2,}$, \\ ${ }^{1}$ Department of Radiology, Shahid Sadoughi University of Medical Sciences and Health Services, Yazd, Iran \\ ${ }^{2}$ Faculty of Medical, Shahid Sadoughi University of Medical Sciences and Health Services, Yazd, Iran
}

Email address:

Sobhan.mo@gmail.com (M. Sobhan),dr.samiee@gmail.com (M. Samiee), yadollah.asgary@gmail.com (Y. Asgari), mansour13662818@gmail.com (M. Ahmadi)

${ }^{*}$ Corresponding author

\section{To cite this article:}

Mohammad Sobhan, Majid Samiee, Yadollah Asgari, Mansour Ahmadi. Incidental Findings of the Lumbar Spine at MRI in Patients Diagnosed with Discopathy. International Journal of Medical Imaging. Vol. 4, No. 5, 2016, pp. 44-47. doi: 10.11648/j.ijmi.20160405.12

Received: August 6, 2016; Accepted: August 19, 2016; Published: September 8, 2016

\begin{abstract}
Background and objectives: the present study is an attempt to investigate the prevalence and type of lumbar spinal MRI incidental findings in patients diagnosed with discopathy. Materials and Methods: This cross-sectional study was conducted from 26/09/2012 to 14/01/2013 on MRI findings of 444 patients clinically diagnosed with Discopathy and approved MRI reports. A Radiologist studied the MRI results in terms of single vertebral hemangioma, multiple vertebral hemangiomas, kidney cyst, liver cyst and Perineural cyst. Data were collected and analyzed through Fisher Exact test. Results: 73 patients (16\%) out of a total of 444 patients (215 males (48.4\%) and 229 females (51.6) with a mean age of 42 years and age range of 13 to 87 years), provided incidental findings. The frequency of single vertebral hemangioma, multiple vertebral hemangioma, kidney cysts, liver cysts and Perineural cyst was 31 cases (7.0\%), 11 cases (2.5\%), 13 cases $(2.9 \%), 2$ cases $(0.5 \%)$ and 3 cases $(0.7 \%)$ respectively. There was no significant correlation between the frequency distribution of findings in terms of sex (P-value $=0.08)$, but there was a significant correlation between the frequency distribution of findings in terms of age $(\mathrm{P}$-value $=0.006)$. Conclusion: incidental findings are fairly common findings in the MRI of patients with discopathy. There is a significant correlation between the frequency distributions of these findings and the patients' age, however, no correlation was observed between these findings and patients' sex.
\end{abstract}

Keywords: Incidental Findings, Lumbar, Magnetic Resonance Imaging

\section{Introduction}

Back pain is one of the major public health problems. Based on previous studies, back pain is the second leading cause of job absenteeism after cold. About 60 to 80 percent of adults experience back pain in their lifetime. The annual Medical expenses caused by back pain reach to $\$ 50$ billion to more than $\$ 100$ billion. Many factors because back pain and these factors include congenital and degenerative spinal stenosis, neoplasm, infectious and inflammatory diseases [1].

Discopathy is one of the major causes of back pain. This disease requires gradual changes in the components of the disk. Inter-vertebral Disc is made of three major components, including the Nucleus Pulposus, Annulus Fibrosus and the end plate. The Nucleus Pulposus forms the gelatin part of the Inter-vertebral Disc. This Nucleus consists of $90 \%$ water and delicate strands of fiber. Fibrous ring surrounds the Nucleus Pulposus and connects the cartilaginous end plate of upper and lower surface of the vertebral bodies to the inter-vertebral discs. The inter-vertebral discs components optimize the mechanical stress exerted on the spine [2].

Inter-vertebral disc damages are more common in the Lumbosacral area at the end of the spine that is under more pressure. Lumbosacral discopathy symptoms include back pain, pain behind the knee, foot side pain, sensory disorders such as tingling, burning, unilateral cramping of lower limb, perineal numbness, as well as urinary, fecal and sexual disorders. Common complications of Discopathy include 
neurological disorders and severe pain and limited physical movements that in some cases can lead to reduced quality of life and even depression [3].

The advent of magnetic resonance imaging (MRI) led to an outstanding development in diagnosis of disease and consequently in treatment of spinal problems. The possibility of havibng multi-dimensional view of anatomy and high-resolution images of soft tissue and the ability to use different sequences in MRI, allow for detailed view of the disk, Fat, nerves, ligaments and bone without the need for injection of contrast agent or exposure to radiation. That's why today MRI, as the best primary test in patients with back pain, is replaced by computerized tumor scan (CT scan) [4].

In fact, incidental findings are the findings that are obtained in an unrelated investigation but are of great clinically importance. These findings could range from normal variants to life-threatening issues which may affect the quality and quantity of life in patients. These findings may in some cases be of more clinical value compared to the issue that has caused patients to have MRI tests. These findings cause clinical and behavioral concerns in patients for seeking treatments on the one hand, and may even be life-threatening and impose costs on the individual and their health system on the other hand. Obtaining information on the prevalence of these findings, on how to manage them and deal with them, their impact on patients' lives, and the impact of these findings on the imaging techniques, notification of patients about the risks and prevalence of normal variants can be of great importance [5-7].

In a study on lawsuits against radiologists in America during 1985-1998, showed that $5 \%$ of condemnations have been due to the failure of radiologists to detect these findings [8].

\section{Materials and Methods}

This is a cross-sectional study on a population of 597 patients who underwent MRI during 26/09/2012 to $14 / 01 / 2013$ due to back pain and a sample of 444 patients clinically diagnosed with Discopathy whose disease was already approved by MRI reports was included in this study. In order to implement this project, the lumbar spine specialists were required to mention (in the application form) their clinical diagnosis of patients disease based on the criteria contained in the References (the same diagnostic criteria), before referring their patients to the Shahid Sadoughi hospital for MRI. MRI tests were examined by a radiologist with sufficient experience in the field of spine MRI and the patients with MRI findings associated with Discopathy were included in the study. The Discopathy- associated findings in this study included Disc Bulging, Disc Herniation that is divided into two subcategories: Disc Protrusion, and Disc Extrusion. The MRIs were reexamined by the radiologist to evaluate the frequency of MRI incidental findings. Incidental findings in this study include: single vertebral hemangioma, multiple vertebral hemangioma, liver cyst, kidney cyst and Perineural cysts.
Vertebral hemangioma can be detected when increased signal intensity in $\mathrm{T} 1 \mathrm{~W}$ is followed by a significant increase in signal intensity in $\mathrm{T} 2 \mathrm{~W}$ and the vertebral bodies has a mottled appearance. Perineural cyst is defined as dilatation in the sheath of the nerves root protuberating out of the sacral region of the spinal cord [9]. Liver cyst in MRI can be detected by Hypointence in T1W and Hyperintence in the T2W. Kidney cysts is detected by reduced signal intensity in T1W and increased signal intensity in $\mathrm{T} 2 \mathrm{~W}[2]$.

All MRI imaging actions were performed by the Simens Magnetom Avanto device with 1.5 Tesla magnet in the Shahid Sadughi hospital of Yazd. The routine protocol for the lumbar spine imaging in this hospital included Sagittal T1W (TR: 520ms, TE: $11 \mathrm{~ms}$, Matrix: $320 \times 240$, FOV: $33 \mathrm{~cm}$ ), Sagittal T2W (TR: $3500 \mathrm{~ms}$, TE: 90ms, Matrix: $384 \times 288$, FOV: $33 \mathrm{~cm}$ ) and Axial T2W (TR: 2570ms, TE103ms, Matrix: $384 \times 226$, FOV: $23 \mathrm{~cm}$ ). The sequences were provided in $4 \mathrm{~mm}$ thick sections and none of them included Fat. Sat.

The patients' information including their age, gender and the incidental findings were analyzed using the spss 17 software. The frequency of findings was measured using descriptive statistics and the age and sex groups were compared based on the Fisher Exact test.

\section{Results}

229 (51.6) patients were female and 215 (48.4) patients were male. The patients were in the age range of 13 to 87 years and their Mean age was $42 \pm 14.2$.

The Age distribution of patients is shown in Figure 1. Discopathy was most frequent in the $30-60$ years old age group $(64.2 \%)$.

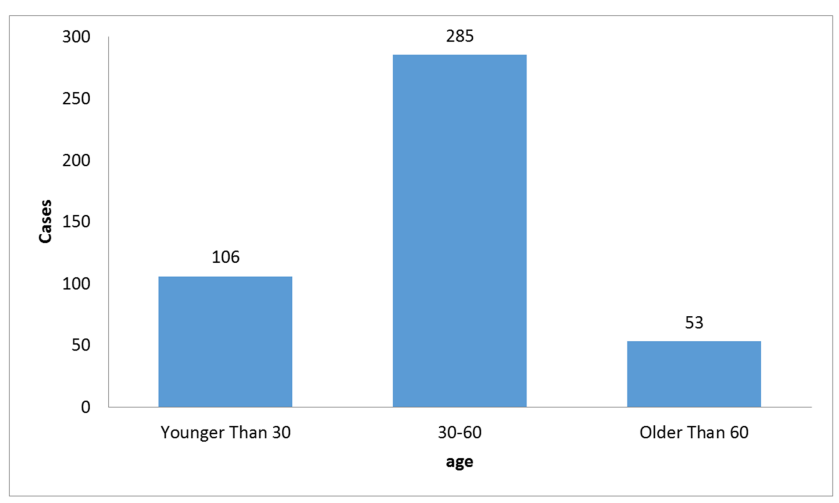

Figure 1. Age Distribution of patients diagnosed with discopathy.

Table 1. Frequency Distribution of concomitant findings in patients with discopathy.

\begin{tabular}{lll}
\hline Incidental Findings & & \\
\hline Without Incidental Findings & 384 & 86.5 \\
Single Vertebral Hemangioma & 31 & 7.0 \\
Multiple Vertebral Hemangioma & 11 & 2.5 \\
Renal Cyst & 13 & 2.9 \\
Liver Cyst & 2 & 0.5 \\
Perineural Cyst & 3 & 0.7 \\
Total & 444 & 100 \\
\hline
\end{tabular}


73 patients $(16 \%)$ provided incidental findings. single vertebral hemangioma was the most frequent finding $(7.0 \%)$, and kidney cysts $(2.9 \%)$ and multiple vertebral hemangiomas $(2.5 \%)$ ranked second and third respectively. Table 1 shows the distribution of incidental findings in patients with Discopathy.

In this study, no significant correlation was observed between patient's sex and incidental findings $(\mathrm{P}>0.05)$. However, a significant correlation was observed between the frequency distribution of incidental findings in terms of age in patients diagnosed with discopathy $(\mathrm{P}<0.05)$. The frequency of incidental findings in different age groups is provided in Table 2.

Table 2. Frequency Distribution of incidental findings in different age groups.

\begin{tabular}{|c|c|c|c|c|c|c|c|}
\hline Age (y) & $\begin{array}{l}\text { Without Incidental } \\
\text { Findings }\end{array}$ & $\begin{array}{l}\text { Single Vertebral } \\
\text { Hemangioma }\end{array}$ & $\begin{array}{l}\text { Multiple Vertebral } \\
\text { Hemangioma }\end{array}$ & $\begin{array}{l}\text { Renal } \\
\text { cyst }\end{array}$ & $\begin{array}{l}\text { Liver } \\
\text { Cyst }\end{array}$ & $\begin{array}{l}\text { Perineural } \\
\text { Cyst }\end{array}$ & Total \\
\hline \multicolumn{8}{|c|}{ No. $(\%)$ of patients } \\
\hline$\leq 29$ & $95(89.6)$ & $4(3.8)$ & $0(0.0)$ & $3(2.8)$ & $2(1.9)$ & $2(2.9)$ & $106(100)$ \\
\hline $30-59$ & $248(87.0)$ & $23(8.1)$ & $7(2.5)$ & $6(2.1)$ & $0(0.0)$ & $1(0.4)$ & $285(100)$ \\
\hline$\geq 60$ & $41(77.4)$ & $4(7.5)$ & $4(7.5)$ & $4(7.5)$ & $0(0.0)$ & $0(0.0)$ & $53(100)$ \\
\hline Total & $384(86.5)$ & $31(7.0)$ & $11(2.5)$ & $13(2.9)$ & $2(0.5)$ & $3(0.7)$ & $444(100)$ \\
\hline
\end{tabular}

\section{Discussion and Conclusion}

Incidental findings is a kind of asymptomatic finding that is detected when patient are examined for other reasons. The impact of incidental findings on patients' health is not clear. However, it should be noted that incidental findings can in some cases be far more important than the problem or which the patients are examined. With the increasing use of MRI, the frequency of these findings is increasing as well. In the study conducted through MRI, The frequency of incidental findings outside the spinal cord was equal to $68.6 \%$, whereas the frequency obtained through CT scan was equal to $40 \%$ [7].

Perineural cyst is meningeal dilation of the sheath in the dorsal base of the spine that is connected to the subarachnoid space. These cysts are asymptomatic in most people. When these cysts develop they start to exert some pressure on the adjacent nerve fibers and causes symptoms such as pain, weakness and sensory dysfunction. The frequency of Perineural cyst in this study was $0.7 \%$. According to the literature, the frequency of these cysts are reported 1-5\% [10, 11].

Vertebral Hemangioma is a benign vascular tumor of the vertebral body. Although this tumor is a common occurrence in the vertebral spine, it is only symptomatic in $1 \%$ of cases. In the study conducted by Barzin and Maleki, the hemangioma frequency was $10-27 \%$ in the autopsy reports. This frequency was slightly higher in older age groups and among women. In the present study, the frequency of vertebral hemangioma was $9.5 \%[12]$.

Simple liver cysts are lesions containing a clear fluid that is not associated with hepatic biliary tree. In the study conducted by Quattrocchi et al, the frequency of liver cysts was $0.2 \%$ [7]. In the study conducted by Tuncel et al. the frequency of these cysts was $0.15 \%$ [13]. The frequency of liver cysts was $0.5 \%$. In the present study. In the studies conducted by Barret et al and Devine et al, to investigate the incidental findings by CT scan, the prevalence of liver cysts was $6.3 \%$ and $4.6 \%$, respectively $[14,15]$. This difference could be due to lower number of liver cuts in lumbar MRI compared to the number of cuts in the abdominal and pelvic CT scan.

Simple renal cysts usually occur in normal kidneys and become more prevalent as people grow older. They usually have no symptoms and rarely need treatment. In a study conducted by Ciezanowski et al. Prevalence of kidney cysts was $25.1 \%$ [16]. Prevalence of kidney cyst was $2.9 \%$ in the present study. This difference could be due to the fact that the $1-1.5 \mathrm{~cm}$ cysts are not reported in our study. In the study conducted by Tuncel et al kidney cysts larger than $1.5 \mathrm{~cm}$ were reported and the prevalence of kidney cyst was $6.4 \%$ [13]. This difference could be due to lower mean age in our study.

Incidental findings are fairly common findings in the MRI of patients with Discopathy. There is a significant correlation between The Distribution frequency of these findings and patients age, however no correlation has been observed between these findings and patients' sex. Obtaining information on the prevalence of these findings can greatly affect the techniques used to manage them and deal with them, their impact on patients' lives, the impact of these findings on the imaging techniques, notification of patients about the risks and prevalence of normal variants.

\section{References}

[1] Frymoyer JW, Cats-Baril WL. An overview of the incidences and costs of low back pain. Orthop Clin North Am. 1991; 22 (2): 263-71.

[2] Haaga JR. CT and MRI of the whole body. 5th ed. Philadelphia, PA: Mosby/Elsevier; 2009.

[3] Dzierżanowski M, Wrzecion K, Słomko W, Radzimińska A, Kaźmierczak U, Strojek $K$, et al. Discopathy of the lumbar-sacral segment and its influence on sexual dysfunction. Adv Clin Exp Med. 2012; 22 (1): 93-100.

[4] Eun SS, Lee HY, Lee SH, Kim KH, Liu WC. MRI versus CT for the diagnosis of lumbar spinal stenosis. J Neuroradiol. 2012; 39 (2): 104-9.

[5] Wagner SC, Morrison WB, Carrino JA, Schweitzer ME, Nothnagel H. Picture archiving and communication system: effect on reporting of incidental findings. Radiology. 2002; 225 (2): 500-5.

[6] Green L. PACS: effect on incidental findings. Radiol Manage. 2004; 26 (1): 26-9. 
[7] Quattrocchi CC, Giona A, Di Martino AC, Errante Y, Scarciolla L, Mallio CA, et al. Extra-spinal incidental findings at lumbar spine MRI in the general population: a large cohort study. Insights Imaging. 2013; 4 (3): 301-8.

[8] Brenner RJ, Lucey LL, Smith JJ, Saunders R. Radiology and medical malpractice claims: a report on the practice standards claims survey of the Physician Insurers Association of America and the American College of Radiology. AJR Am J Roentgenol. 1998; 171 (1): 19-22.

[9] Park H-J, Jeon Y-H, Rho M-H, Lee E-J, Park N-H, Park S-I, et al. Incidental findings of the lumbar spine at MRI during herniated intervertebral disk disease evaluation. AJR Am J Roentgenol. 2011; 196 (5): 1151-5.

[10] Nabors MW, Pait TG, Byrd EB, Karim NO, Davis DO, Kobrine AI, et al. Updated assessment and current classification of spinal meningeal cysts. J Neurosurg. 1988; 68 (3): 366-77.

[11] Voyadzis J-M, Bhargava P, Henderson FC. Tarlov cysts: a study of 10 cases with review of the literature. J Neurosurg. 2001; 95 (1): 25-32.
[12] Barzin M, Maleki I. Incidence of vertebral hemangioma on spinal magnetic resonance imaging in Northern Iran. Pak J Biol Sci. $2009 ; 12$ (6): 542-4.

[13] Tuncel SA, Cagli B, Tekatas A, Kirici MY, Unlu E, Genchellac H. Extraspinal Incidental Findings on Routine MRI of Lumbar Spine: Prevalence and Reporting Rates in 1278 Patients. Korean J Radiol. 2015; 16 (4): 866-73.

[14] Barrett TW, Schierling M, Zhou C, Colfax JD, Russ S, Conatser $\mathrm{P}$, et al. Prevalence of incidental findings in trauma patients detected by computed tomography imaging. Am J Emerg Med. 2009; 27 (4): 428-35.

[15] Devine AS, Jackson CS, Lyons L, Mason JD. Frequency of incidental findings on computed tomography of trauma patients. West J Emerg Med. 2010; 11 (1): 24-7.

[16] Cieszanowski A, Maj E, Kulisiewicz P, Grudzinski IP, Jakoniuk-Glodala K, Chlipala-Nitek I, et al. Non-contrast-enhanced whole-body magnetic resonance imaging in the general population: the incidence of abnormal findings in patients 50 years old and younger compared to older subjects. PLoS One. 2014; 9 (9): e107840. 